\title{
Correction to: Analysis of the SRISK measure and its application to the Canadian banking and insurance industries
}

\author{
Thomas F. Coleman ${ }^{1}$ - Alex LaPlante ${ }^{2}$. Alexey Rubtsov ${ }^{2}$
}

Published online: 15 September 2018

(c) Springer-Verlag GmbH Germany, part of Springer Nature 2018

\section{Correction to: Ann Finance https://doi.org/10.1007/s10436-018-0326-3}

In the original publication, Table 6 was incorrect. The correct version of Table 6 is given for your reading. The original article has been corrected.

The original article can be found online at https://doi.org/10.1007/s10436-018-0326-3.

$\bowtie$ Alexey Rubtsov

alexeyVrubtsov@gmail.com

Thomas F. Coleman

tfcoleman@uwaterloo.ca

Alex LaPlante

alaplante@globalriskinstitute.org

1 Waterloo Research Institute in Insurance, Securities, and Quantitative Finance (WatRISQ),

University of Waterloo, 200 University Avenue West, Waterloo, ON N2L 3G1, Canada

2 Global Risk Institute in Financial Services, 55 University Avenue, Suite 180, Toronto, ON M5J 2H7, Canada 
Table 6 Change in SRISK due to Debt, LRMES, and Equity changes, in million CAD. (Dec. 2015-Jun. 2016)

\begin{tabular}{|c|c|c|c|c|c|c|c|}
\hline & & TD & Scotiabank & BMO & $\mathrm{RBC}$ & CIBC & Total \\
\hline \multirow[t]{7}{*}{$\Delta(D)$} & 31-Dec-14 & 2799 & 1004 & 54 & 1926 & 691 & 6473 \\
\hline & 31-Mar-15 & 8493 & 3318 & 6108 & 10,742 & 2222 & 30,883 \\
\hline & 30-Jun-15 & -3613 & -1086 & -2837 & -4151 & -482 & $-12,168$ \\
\hline & $30-$ Sep-15 & 4791 & 1791 & 2766 & 3701 & 1309 & 14,359 \\
\hline & 31-Dec-15 & 308 & -531 & -2384 & -976 & 373 & -3210 \\
\hline & 31-Mar-16 & 4842 & 4571 & 4178 & 9015 & 1148 & 23,754 \\
\hline & 30-Jun-16 & -3371 & -1712 & -1143 & -3617 & -52 & -9896 \\
\hline$\sum \Delta(\mathrm{D})$ & & 14,250 & 7355 & 6742 & 16,639 & 5210 & 50,196 \\
\hline \multirow[t]{7}{*}{$\Delta($ Risk) } & 31-Dec-14 & 1839 & 923 & -694 & 376 & 1555 & 3999 \\
\hline & 31-Mar-15 & -3052 & 1928 & -808 & 2472 & -708 & -169 \\
\hline & 30-Jun-15 & 2660 & -1997 & 1277 & -2433 & -74 & -566 \\
\hline & $30-$ Sep-15 & 5939 & 4359 & 3053 & 4554 & 2963 & 20,868 \\
\hline & 31-Dec-15 & 3546 & 3646 & 654 & 7057 & 1081 & 15,985 \\
\hline & 31-Mar-16 & $-10,752$ & -6397 & -2524 & -9241 & -2092 & $-31,006$ \\
\hline & 30-Jun-16 & 6735 & 1557 & 193 & 3552 & 1894 & 13,931 \\
\hline$\sum \Delta$ (Risk) & & 6914 & 4019 & 1151 & 6337 & 4621 & 23,042 \\
\hline \multirow[t]{7}{*}{$\Delta(E)$} & 31-Dec-14 & -407 & 2666 & 55 & -241 & 208 & 2279 \\
\hline & 31-Mar-15 & 1635 & 2728 & 3144 & 4005 & 2294 & 13,805 \\
\hline & 30-Jun-15 & 1437 & -810 & 1043 & -35 & -82 & 1554 \\
\hline & $30-$ Sep-15 & 552 & 4863 & 648 & 2620 & -1068 & 7616 \\
\hline & 31-Dec-15 & -2039 & 2380 & -2211 & -2370 & 1216 & -3024 \\
\hline & 31-Mar-16 & -2114 & -5936 & -374 & -772 & -1342 & $-10,538$ \\
\hline & 30-Jun-16 & 657 & 103 & -1386 & -1614 & -22 & -2261 \\
\hline$\sum \Delta(\mathrm{E})$ & & -280 & 5993 & 918 & 1593 & 1206 & 9431 \\
\hline
\end{tabular}

